\title{
Electron Spectrum and Tunneling Current of the Toroidal and Helical Graphene Nanoribbon-Quantum Dots Contact
}

\author{
Mikhail B. Belonenko, ${ }^{1,2}$ Nikolay G. Lebedev, ${ }^{3}$ Alexander V. Zhukov, ${ }^{4,2}$ \\ and Natalia N. Yanyushkina ${ }^{3}$ \\ ${ }^{1}$ Volgograd Institute of Business, Yuzhno-Ukrainskaya Street 2, 400048 Volgograd, Russia \\ ${ }^{2}$ Scientific Department, Entropique Inc., London, ON, Canada N6J 352 \\ ${ }^{3}$ Volgograd State University, University Avenue 100, 400062 Volgograd, Russia \\ ${ }^{4} M^{2} \mathrm{NeT}$ Lab, Wilfrid Laurier University, Waterloo, ON, Canada N2L 3C5 \\ Correspondence should be addressed to Mikhail B. Belonenko, mbelonenko@yandx.ru
}

Received 29 March 2011; Accepted 17 April 2011

Academic Editors: B. Coasne and S. Maksimenko

Copyright () 2011 Mikhail B. Belonenko et al. This is an open access article distributed under the Creative Commons Attribution License, which permits unrestricted use, distribution, and reproduction in any medium, provided the original work is properly cited.

We study the electron spectrum and the density of states of long-wave electrons in the curved graphene nanoribbon based on the Dirac equation in a curved space-time. The current-voltage characteristics for the contact of nanoribbon-quantum dot have been revealed. We also analyze the dependence of the specimen properties on its geometry.

\section{Introduction}

The problem of modified graphene properties attracts a considerable attention of researchers $[1,2]$ because the "pure" graphene has no energy gap in the band structure and, therefore, the creation of different structures (e.g., analogs of transistors) is extremely difficult. However, the situation becomes more promising when various modifications of the specimen are introduced. As an example, we consider the modified graphene, for example, graphene nanoribbon, which have quantized electron energy spectrum due to the limited space in one dimension, which in turn can lead to the formation of the energy gap. Furthermore, we know that the graphene has a naturally wave-like curved surface due to the instability of the planar structure of its sheets $[3,4]$. All of the above reasons have stimulated the study of different modifications of a curved graphene $[5,6]$. The long-wave approximation, which is widely used to describe the properties of electrons in graphene, leads to an analog of the Dirac equation, which in turn makes it easy to produce generalization to the case when the graphene surface is curved. Note that in this case the degeneracy in the Dirac points is removed and therefore it becomes possible to create various structures with different band gaps. Consideration of the Dirac equation for curved graphene [5] also shows a change in the density of states of electrons and, therefore, it is possible to change the whole set of electrical characteristics of the graphene sample. Apparently, the easiest way to experimentally verify the changes in the density of states is to study the tunneling current [7], for example, through the contact with quantum dots. Quantum dots are still rather "young" objects of study, but their use in various fields of science and technology is obviously extremely promising (from the design of lasers and new generation displays to building qubits) [8].

\section{Basic Equations and Spectrum of Electrons}

We consider the graphene nanoribbon, which is curved along the toroidal and the helical surfaces, as presented in Figure 1.

Properties of electrons in the graphene nanoribbons in the long-wave approximation in the vicinity of the Dirac point will be described on the basis of the Dirac equation generalized for the case of a curved space-time [9]:

$$
\gamma^{\mu}\left(\partial_{\mu}-\Omega_{\mu}\right) \Psi=0
$$




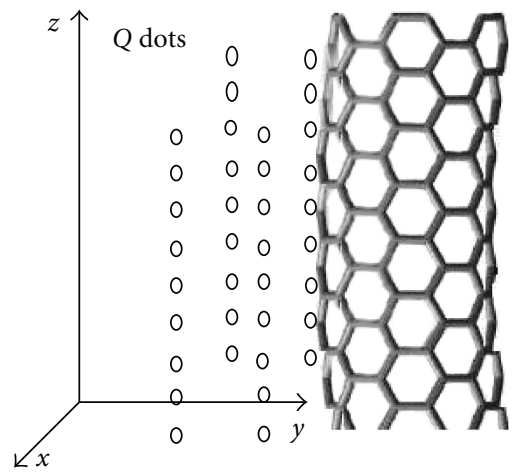

(a)

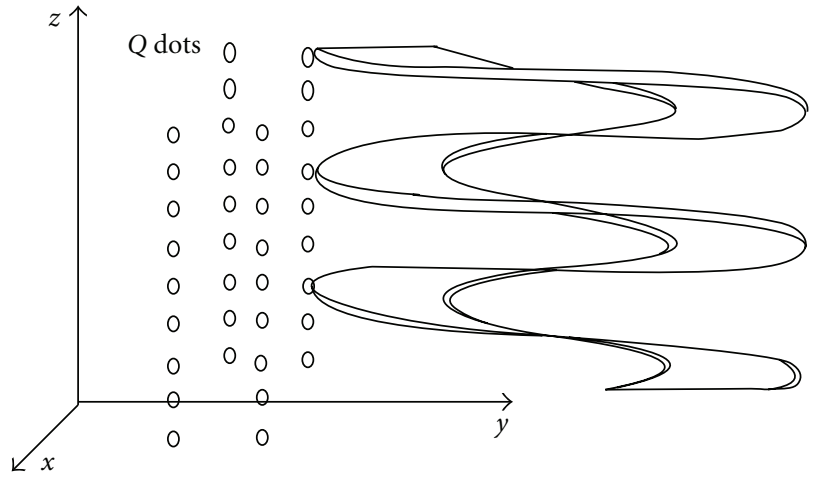

(b)

FIGURE 1: Geometry of a problem; (a) toroidal nanoribbon, (b) helical nanoribbon.

Here and below the repeated indices assume summation; $\partial_{\mu}$ is the partial derivative with respect to coordinate $\mu, \Omega_{\mu}$ is the component of the spin connection, and $\Psi=\left(\begin{array}{l}\phi \\ \varphi\end{array}\right)$ is the wave function (column vector) consisting of wave functions describing the electrons from different sublattices near the Dirac point.

As is known $[9,10]$, if we are given the metric tensor

$$
\begin{gathered}
d s^{2}=g_{\alpha \beta} d x^{\alpha} d x^{\alpha}, \\
g_{\alpha \beta} g^{\beta \gamma}=\delta_{\alpha}^{\gamma}
\end{gathered}
$$

$\left(\delta_{\alpha}^{\gamma}\right.$ : delta Kronecker symbol) then, we can define the field frames (tetrads):

$$
\begin{gathered}
g_{\alpha \beta}=e_{\alpha}^{a} e_{\beta}^{b} \eta_{a b}, \\
g^{\alpha \beta}=e_{a}^{\alpha} e_{b}^{\beta} \eta^{a b}, \\
\eta_{a b} \eta^{b c}=\delta_{a}^{c},
\end{gathered}
$$

where, for the two-dimensional curved surface, we have $\eta_{a b}=\operatorname{diag}(1,-1,-1)$. Then,

$$
\begin{gathered}
\Omega_{\mu}=\frac{1}{4} \gamma_{a} \gamma_{b} e_{\lambda}^{a} g^{\lambda \sigma}\left(\partial_{\mu} e_{\sigma}^{b}-\Gamma_{\mu \sigma}^{\lambda} e_{\lambda}^{b}\right), \\
\Gamma_{\mu \sigma}^{\lambda}=\frac{1}{2} g^{\lambda \nu}\left(g_{\sigma \nu, \mu}+g_{\nu \mu, \sigma}-g_{\mu \sigma, \nu}\right), \\
\gamma^{\mu}=e_{a}^{\mu} \gamma_{a} .
\end{gathered}
$$

Using the torus and the helical parameterization

$$
\begin{gathered}
x=\left(R+r \cos x_{1}\right) \cos x_{2}, \\
y=\left(R+r \cos x_{1}\right) \sin x_{2}, \\
z=r \sin x_{1}, \\
x=x_{1} \cos x_{2}, \\
y=x_{1} \sin x_{2}, \\
z=h \cdot x_{2},
\end{gathered}
$$

we find that the metrics on the torus surface and the helicoid is

$$
\begin{gathered}
d s^{2}=d x_{0}^{2}-r^{2} d x_{1}^{2}-\left(R+r \cos x_{1}\right)^{2} d x_{2}^{2}, \\
d s^{2}=d x_{0}^{2}-d x_{1}^{2}-\left(h^{2}+x_{1}^{2}\right) d x_{2}^{2} .
\end{gathered}
$$

Note that all the Christoffel symbols are equal to zero, except $\Gamma_{12}^{2}$ and $\Gamma_{22}^{1}$. For the torus, we have $\Omega_{0}=0, \Omega_{1}=0$, $\Omega_{2}=(1 / 2) \gamma_{1} \gamma_{2} f^{\prime} / r\left(f=R+r \cos x_{1}\right.$, and $\left.f^{\prime}=\partial f / \partial x_{1}\right)$, while in the case of helicoid $\Omega_{0}=0, \Omega_{1}=0$, and $\Omega_{2}=$ $(1 / 2) \gamma_{1} \gamma_{2}\left(x_{1} /\left(h^{2}+x_{1}^{2}\right)^{1 / 2}\right)$.

Choosing $\gamma_{0}=\sigma_{3}, \gamma_{1}=-i \sigma_{2}$, and $\gamma_{2}=-i \sigma_{1}$, where $\sigma$ are the Pauli matrices, we obtain the following system of equations:

$$
\begin{gathered}
V_{F}^{-1} \partial_{t} \varphi=-\frac{1}{r^{2}} \partial_{x_{1}} \Psi-\frac{i}{f^{2}} \partial_{x_{2}} \Psi+\frac{f^{\prime}}{2 f^{2} r} \Psi, \\
V_{F}^{-1} \partial_{t} \varphi=-\frac{1}{r^{2}} \partial_{x_{1}} \varphi+\frac{i}{f^{2}} \partial_{x_{2}} \varphi+\frac{f^{\prime}}{2 f^{2} r} \varphi, \\
V_{F}^{-1} \partial_{t} \varphi+\partial_{x_{1}} \Psi+\frac{i}{h^{2}+x_{1}^{2}} \partial_{x_{2}} \Psi-\frac{x_{1}}{2\left(h^{2}+x_{1}^{2}\right)^{3 / 2}} \Psi=0, \\
-V_{F}^{-1} \partial_{t} \Psi-\partial_{x_{1}} \varphi+\frac{i}{h^{2}+x_{1}^{2}} \partial_{x_{2}} \varphi-\frac{x_{1}}{2\left(h^{2}+x_{1}^{2}\right)^{3 / 2}} \varphi=0 .
\end{gathered}
$$

Here, $V_{F}$ is the Fermi velocity for planar graphene, $\partial_{0}=$ $V_{F}^{-1} \partial_{t}$. Note that since the metric (6) admits two Killing vectors corresponding to translations along $x_{0}, x_{2}$, the solution (7) can be found in the form

$$
\left(\begin{array}{c}
\varphi \\
\Psi
\end{array}\right) \longrightarrow\left(\begin{array}{c}
\varphi\left(x_{1}\right) \\
\Psi\left(x_{1}\right)
\end{array}\right) e^{i E t-i k x_{2}}
$$


which finally gives

$$
\begin{aligned}
\Psi^{\prime \prime}= & \left(-\frac{E^{2} r^{4}}{V_{f}^{2}}+\frac{k_{n}^{2} r^{4}}{f^{4}}\right) \Psi+\frac{r f^{\prime}}{2 f^{2}} \Psi^{\prime} \\
& +\left(\frac{2 k_{n} r^{2} f^{\prime}}{f^{3}}+\frac{r f^{\prime \prime}}{2 f^{2}}-\frac{r f^{\prime 2}}{f^{3}}-\frac{r^{2} f^{\prime 2}}{4 f^{4}}\right) \Psi, \\
\Psi^{\prime \prime}= & \left(-\frac{\varepsilon^{2}}{V_{f}^{2}}+\frac{k^{2}}{\left(h^{2}+x_{1}^{2}\right)^{2}}\right) \Psi \\
& +\left(-\frac{k x_{1}}{\left(h^{2}+x_{1}^{2}\right)^{5 / 2}}+\frac{x_{1}^{2}}{4\left(h^{2}+x_{1}^{2}\right)^{3}}\right) \Psi .
\end{aligned}
$$

Note that the wave vector $k$ is found from the boundary conditions at the ends of the nanoribbon. In our particular case, we have chosen the armchair-type ribbon [6] and

$$
k_{n}=\frac{2 \pi}{3 a_{0}}\left(\frac{2 M+1+n}{2 M+1}\right)
$$

where $a_{0}$ is the distance between the atoms in the carbon lattice, $M$ is the number of atoms along nanoribbon axis, and $n$ is the quantum number. We can consider (9) as a Schrodinger equation with perturbation

$$
\begin{gathered}
\hat{V}_{\text {Torus }}=\left[\left(\frac{2 k_{n} r^{2} f^{\prime}}{f^{3}}+\frac{r f^{\prime \prime}}{2 f^{2}}-\frac{r f^{\prime 2}}{f^{3}}-\frac{r^{2} f^{\prime 2}}{4 f^{4}}\right)+\frac{r f^{\prime}}{2 f^{2}} \partial_{x}\right], \\
\hat{V}_{\text {Helicoid }}=\left(-\frac{k x_{1}}{\left(h^{2}+x_{1}^{2}\right)^{5 / 2}}+\frac{x_{1}^{2}}{4\left(h^{2}+x_{1}^{2}\right)^{3}}\right) .
\end{gathered}
$$

In this case, the spectrum of perturbation looks like

$$
E= \pm \sqrt{k_{n}^{2}+k_{y}^{2}}
$$

Expanding the functions in the denominator in Taylor series to the second order, we calculate the first perturbation correction to the spectrum, $\hat{V}_{\text {Torus }}$ and $\hat{V}_{\text {Helicoid }}$ as follows:

$$
E_{1}=\int \Psi^{*} \hat{V} \Psi d x, \quad \Psi_{n}=A \cdot \operatorname{Sin}\left(k_{n} x_{1}\right) .
$$

The integration is performed from 0 to $L=(3 M+1) a_{0}$, and the corrections are as follows:

$$
\begin{aligned}
& E_{1}=\frac{2}{L}\{- \frac{r^{2}}{4 R^{2}} \operatorname{Sin}(L)+\frac{r^{2}}{8 R^{2}}\left(1-\frac{1}{k_{n}}\right) \frac{\operatorname{Sin}\left(2 k_{n}-1\right) L}{\left(2 k_{n}-1\right)} \\
&\left.+\frac{r^{2}}{8 R^{2}}\left(1+\frac{1}{k_{n}}\right) \frac{\operatorname{Sin}\left(2 k_{n}+1\right) L}{\left(2 k_{n}+1\right)}\right\}, \\
& E_{1}=\frac{2}{L}\left\{\left(-\frac{k L^{2}}{4 h^{5}}+\frac{k}{4 h^{5} k_{n}^{2}}+\frac{h^{-2 / 3} L^{3}}{24}\right)\right. \\
&+\operatorname{Sin}\left(2 k_{n} L\right)\left(\frac{k L}{4 h^{5} k_{n}}+h^{-2 / 3}\left(\frac{1}{32 k_{n}^{3}}-\frac{L^{2}}{16 k_{n}}\right)\right) \\
&\left.+\operatorname{Cos}\left(2 k_{n} L\right)\left(\frac{-k}{4 h^{5} k_{n}^{4}}+\frac{h^{-2 / 3} L}{16 k_{n}^{2}}\right)\right\} .
\end{aligned}
$$

The dependence of the perturbations on the atom numbers along the nanoribbons $M$ is presented in Figure 2.

The dependence shown in Figure 2(a) is rather complex, which is associated with the quantization of the electron spectrum in graphene nanoribbons in accordance with (10). It should be noted that the dependence of the energy gap in carbon nanotubes of zigzag type is pretty much similar, which also arises from the quantization of the electron spectrum in the direction along the circumference of the nanotube. The calculations (Figure 2(b)) show that the value of the helicoids parameterization $h$ influences most strongly the correction to the energy (as well as its sign).

\section{Tunneling Characteristics}

The Hamiltonian of the system of electrons can be written in the following form:

$$
H=\sum_{p} E_{p}^{A} a_{p}^{+} a_{p}+\sum_{q} E_{q}^{B} b_{q}^{+} b_{q}+\sum_{p q} T_{p q}\left(a_{p}^{+} b_{q}+b_{q}^{+} a_{q}\right),
$$

where $a_{p}^{+}, a_{p}$ are the electron creation and annihilation operators with momentum $p$ in the carbon nanoribbons, $E_{p}^{A}$ is the electron spectrum of the carbon nanoribbons (12) with taking into account (14), $T_{p q}$ is the matrix element of tunneling operator between $p$ and $q$ states, $b_{q}^{+}, b_{q}$ are the electron creation and annihilation operators with momentum $q$ in a substance which is in a contact with a carbon nanoribbon, and $E_{p}^{B}$ is the electron spectrum of another substance. It should be noted that $p$ and $q$ are multiindices in (15). So, for graphene nanoribbon (further, we consider an arm-chair nanoribbon only), $p=\left(p_{y}, n\right), n=$ $0,1, \ldots, M-1$. Multi-index $q$ is determined by the substance which contacts with carbon nanoribbon and, for example, for quantum dots $q=\left(p_{x}, p_{y}, p_{z}\right)$, whereas for grapheme $q=\left(p_{x}, p_{y}\right)$. A consideration of the external electric field $\vec{E}$ (and choosing $\vec{E}=-(1 / c)(\partial \vec{A} / \partial t))$ can be carried out by the replacement $p \rightarrow p-e A / c$.

Tunneling current is considered to be given by

$$
J=i e \sum_{p q}\left(a_{p}^{+} b_{q}-b_{q}^{+} a_{p}\right) .
$$

With a gauge transformation [11],

$$
\begin{gathered}
a_{p} \longrightarrow S^{-1} a_{p} S, \\
S=\exp \left(i e V t \sum_{p} a_{p}^{+} a_{p}\right),
\end{gathered}
$$

where $V$ is the applied voltage and $e$ is the electron charge. Formally, it is possible to reduce a problem of calculation of the current-voltage characteristics to the calculation of the operator response

$$
J_{t}=i e \sum_{p q}\left(a_{p}^{+} b_{q} e^{i e V t}-b_{q}^{+} a_{q} e^{-i e V t}\right)
$$

on the external influence [11]

$$
H_{t}=\sum_{p q} T_{p q}\left(a_{p}^{+} b_{q} e^{i e V t}+b_{q}^{+} a_{q} e^{-i e V t}\right) .
$$




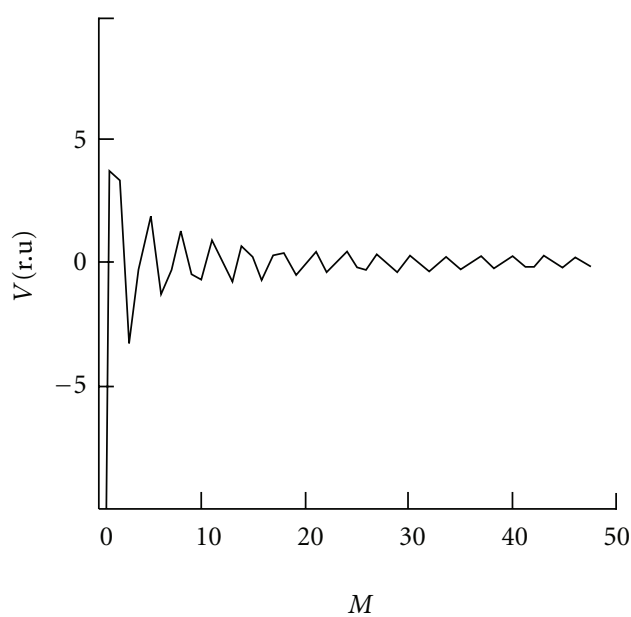

(a)

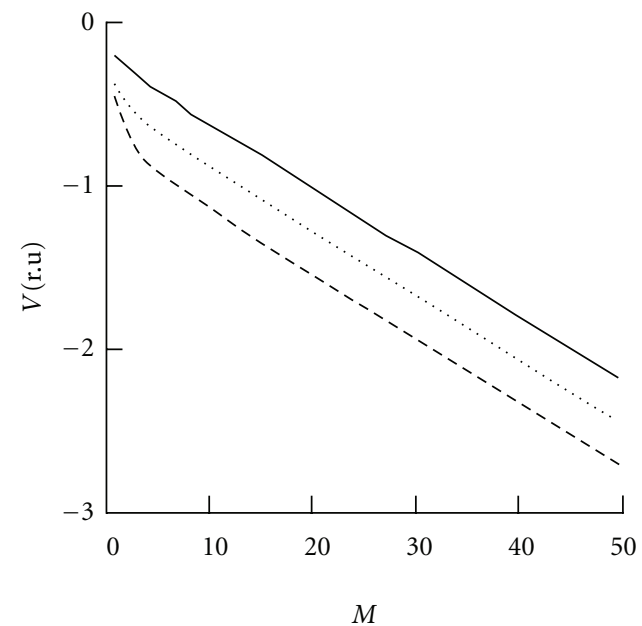

(b)

FIGURE 2: Dependence of the correction to the energy $V$ caused by the perturbation $\hat{V}$ on atoms number along nanoribbon axis $M$ : (a) for torus $(r / R=0.1, n=1)$; (b) for helicoid $(h=1.5)$ : (a) $n=1$, (b) $n=2$, (c) $n=3$.

The solution was obtained within the framework of the Kubo theory:

$$
\begin{gathered}
J=4 \pi e|T|^{2} \int_{-\infty}^{\infty} d E v_{A}(E+e V) \nu_{B}(E)\left(n_{f}(E)-n_{f}(E+e V),\right. \\
\nu_{A}(E)=\sum_{p} \delta\left(E-E_{p}^{A}\right) ; \quad v_{B}(E)=\sum_{q} \delta\left(E-E_{q}^{B}\right),
\end{gathered}
$$

where $\delta(x)$ is the Dirac delta function, $v_{A(B)}(E)$ is the tunneling density of states, and $n_{f}(E)$ is the equilibrium number of fermions with the energy $E$. The approximation of a "rough" contact is hereinafter used, so that $T_{p q}=T$ (this imposes certain restrictions on the contact geometry, i.e., for the case discussed below means that nanoribbon should be perpendicular to the contact material surface). For definiteness, we choose the dispersion law for the graphene nanoribbons given by (12) and (14) and the dispersion law for the quantum dots as the contact material being

$$
E_{q}^{A}=\varepsilon_{0}-\Delta \cos (p),
$$

where $\varepsilon_{0}$ is the electron energy of a quantum well, $\Delta$ is the tunneling integral determined by the overlap of electron wave functions in the adjacent wells, and the momentum $p$ is directed along the axis $Z$.

Equation (20) under study has been solved numerically. The current-voltage characteristic of the contact is presented in Figure 3.

Figure 3 shows the asymmetric behavior of current versus voltage applied to the contact. This is due to both the peculiarities of the electronic structure (density of states) of the metal and graphene nanoribbon and the processes of carrier recombination in the transition contact, which dominate over the thermal processes when $V>$ 0 . The resulting dependence may have important practical applications in the study of nanocontacts and the design

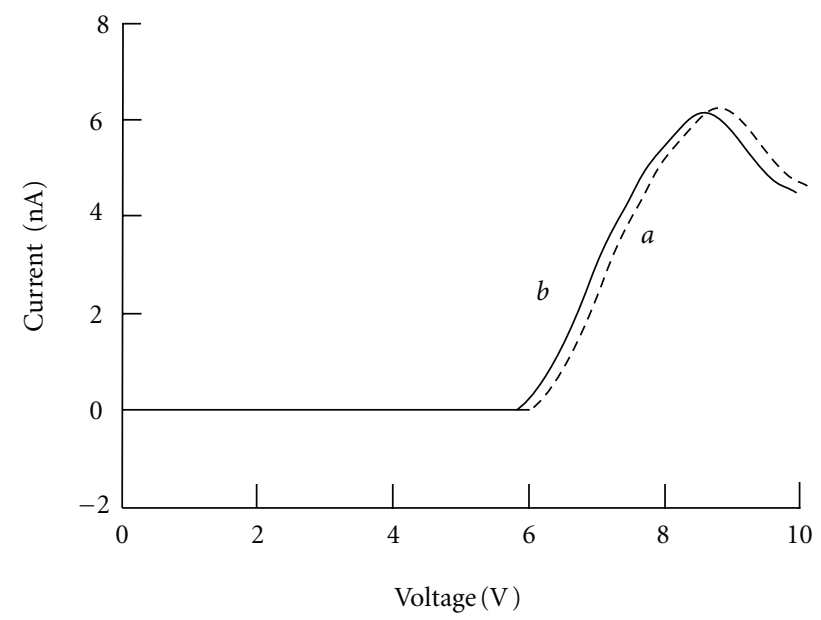

FIgURE 3: The current-voltage characteristic of the contact: curved grapheme nanoribbon-quantum dots: (a) for torus; (b) for helicoid.

of tunnel diodes based on graphene nanoribbons. Also, the region with negative differential resistance was observed for some values of $V$. The presence of such region allows the use of a tunnel diode as a high-speed switch.

\section{Conclusion}

(1) An effective equation, which describes the electrons in a curved graphene nanoribbon and the tunneling contact nanoribbon-quantum dot has been obtained.

(2) The dependence of the electron spectrum on the geometry has been revealed. In general, the first perturbation correction to the spectrum is defined by the atoms number along the nanoribbon axis $M$ and by the parametric coefficient, which describes 
the "curvature" of nanoribbon, $r / R$ (in the torus nanoribbon case), and the step for helical nanoribbon $h$.

(3) The current-voltage characteristics for the contact graphene nanoribbon-quantum dots was constructed; the regions with negative differential conductivity were found.

\section{Acknowledgments}

This work was supported by the Russian Foundation for Basic Research under project no. 08-02-00663 and by the Federal Target Program "Scientific and pedagogical manpower" for 2010-2013. This work was partially supported by NSERC and CRC Program, Canada, and by the Russian Foundation for Basic Research under project no. 11-0297054-r_povolzhè_a.

\section{References}

[1] Y. Zhang, Y. W. Tan, H. L. Stormer, and P. Kim, "Experimental observation of the quantum Hall effect and Berry's phase in graphene," Nature, vol. 438, no. 7065, pp. 201-204, 2005.

[2] S. Stankovich, D. A. Dikin, G. H. B. Dommett et al., "Graphene-based composite materials," Nature, vol. 442, no. 7100, pp. 282-286, 2006.

[3] A. Cortijo and M. A. H. Vozmediano, "Effects of topological defects and local curvature on the electronic properties of planar graphene," Nuclear Physics B, vol. 763, no. 3, pp. 293308, 2007.

[4] A. Cortijo and M. A. H. Vozmediano, "Electronic properties of curved graphene sheets," Europhysics Letters, vol. 77, no. 4, Article ID 47002, 2007.

[5] D. V. Kolesnikov and V. A. Osipov, "Electronic structure of negatively curved graphene," JETP Letters, vol. 87, no. 8, pp. 419-422, 2008.

[6] L. Brey and H. A. Fertig, "Electronic states of graphene nanoribbons studied with the Dirac equation," Physical Review B, vol. 73, no. 23, Article ID 235411, 5 pages, 2006.

[7] M. B. Belonenko, N. G. Lebedev, and N. N. Yanyushkina, "Tunneling through the carbon nanotube/graphene interface exposed to a strong oscillating electric field," Journal of Nanophotonics, vol. 4, Article ID 041670, 2010.

[8] N. Cobayasi, Introduction in Nanotechnology, BINOM, Moscow, Russia, 2007.

[9] M. A. H. Vozmediano, M. I. Katsnelson, and F. Guinea, "Gauge fields in graphene," Physics Reports, vol. 496, no. 4-5, pp. 109$148,2010$.

[10] N. Birrel and P. Devis, Quantum Fields in Curved Space-Time, Moscow: Mir, 1984.

[11] L. S. Levitov and A. V. Shitov, Green's Functions. Tasks with Answers: Fizmatlit, 2003. 

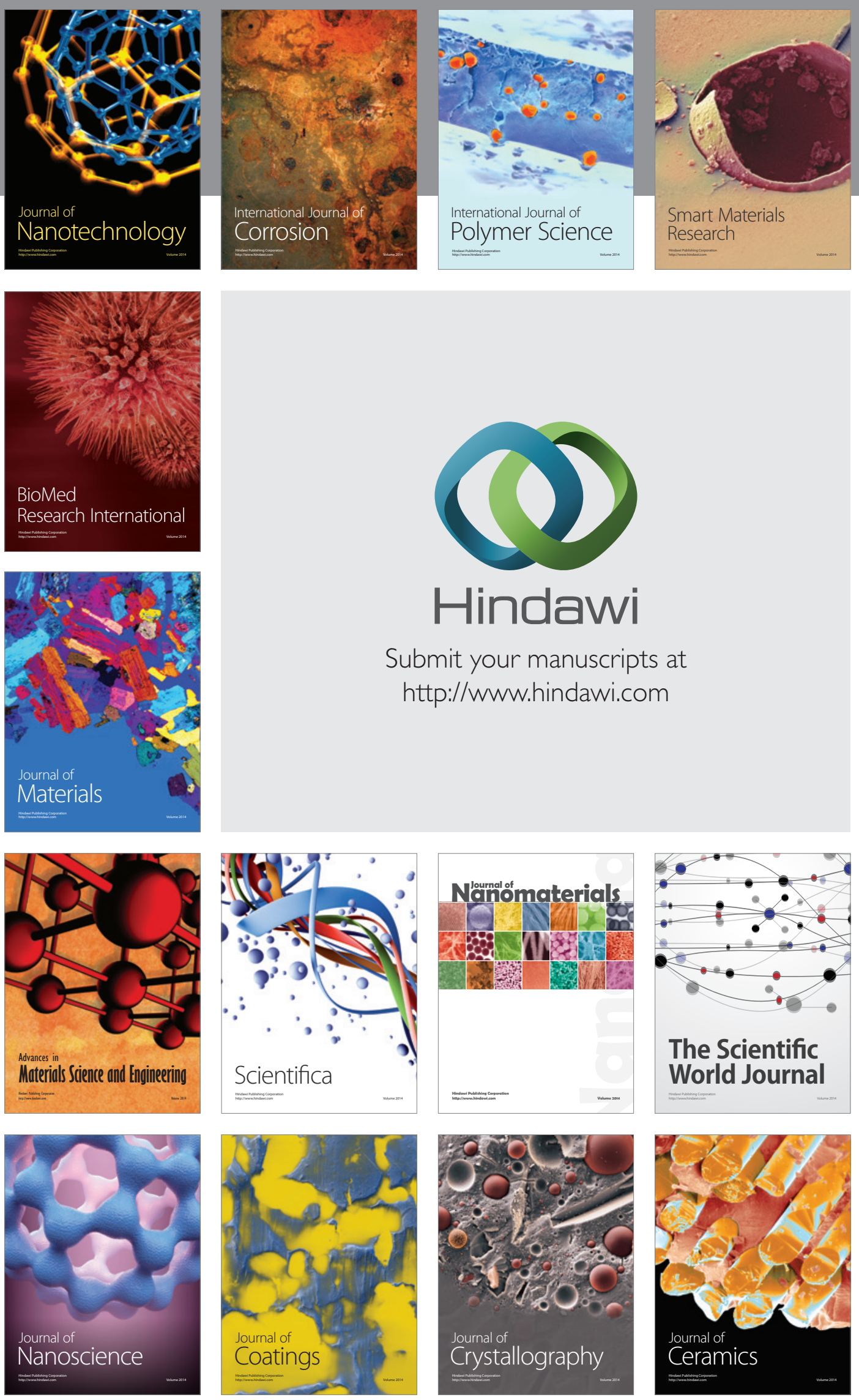

The Scientific World Journal

Submit your manuscripts at

http://www.hindawi.com

\section{World Journal}

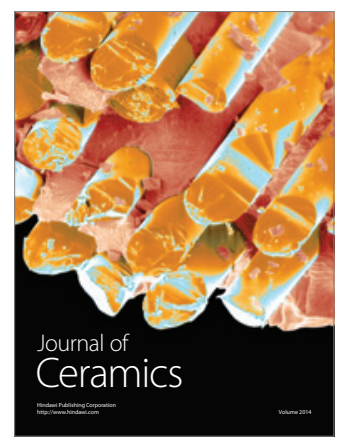

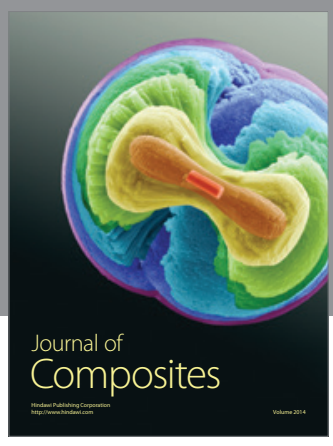
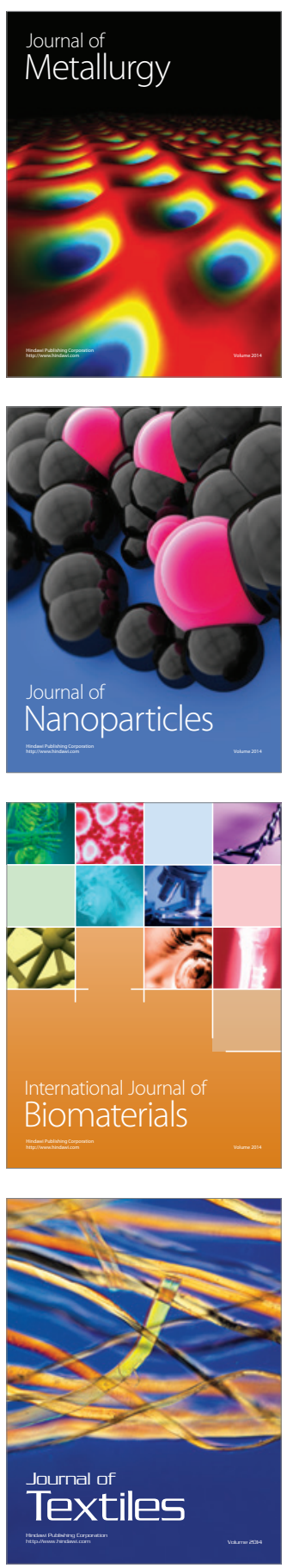